SIT-HEP/TM-58

\title{
A new perspective on supersymmetric inflation
}

\author{
Tomohiro Matsuda 1 \\ Laboratory of Physics, Saitama Institute of Technology, \\ Fusaiji, Okabe-machi, Saitama 369-0293, Japan
}

\begin{abstract}
We consider supersymmetric inflation with the hybrid-type potential. In the absence of the symmetry that forbids Hubble-induced mass terms, the inflaton mass will be as large as the Hubble scale during inflation. We consider gravitational decay of the trigger field as the least decay mode and find that the damping caused by the dissipation can dominate the friction of the inflaton when the heavy trigger field is coupled to the inflaton. The dissipative damping provides a solution to the traditional $\eta$ problem without introducing additional symmetry and interactions. Considering the spatial inhomogeneities of the dissipative coefficient, we find that modulated inflation (modulation of the inflaton velocity) can create significant curvature perturbations.
\end{abstract}

\footnotetext{
${ }^{1}$ matsuda@sit.ac.jp
} 


\section{Introduction and model}

Warm inflation is known as the scenario of dissipative inflaton motion[1]. In this paper we address the generic situation where the inflaton field, which couples to a massive trigger field, dissipates its energy into light fields during inflation without introducing additional interactions. Namely, a trigger field $\chi$ coupled to the inflaton $\phi$ with the interaction $\sim \frac{g^{2}}{2} \phi^{2} \chi^{2}$ obtains large mass $m_{\chi} \sim g \phi$ during inflation, and decays (at least gravitationally) into light matter with a decay rate

$$
\Gamma_{\chi} \simeq \frac{m_{\chi}^{3}}{M_{p}^{2}}
$$

The condition for fast decay $\left(\Gamma_{\chi}>H\right)$ is trivial. We define the critical value $\phi_{*}$ by

$$
\phi_{*} \equiv \frac{\left(H M_{p}^{2}\right)^{1 / 3}}{g}
$$

and find that the decay into light matter $(\chi \rightarrow 2 \psi)$ is efficient for $\phi>\phi_{*}$. For a more concrete argument, we consider the hybrid-type potential with the superpotential

$$
W=g \Phi\left(Q_{+} Q_{-}-M^{2}\right),
$$

where $\Phi$ is the inflaton, and $Q_{ \pm}$are a pair of charged fields (trigger fields) that are responsible for reheating after inflation. For the region $\phi \gg M$, we consider the typical flat potential

$$
V(\phi)=V_{I}+\frac{c_{H}}{2} H^{2}|\phi|^{2}+\left(\frac{\lambda_{n} H}{n M_{p}^{n-3}} \phi^{n}+\text { h.c. }\right)+\left|\lambda_{n}\right|^{2} \frac{|\phi|^{2 n-2}}{M_{p}^{2 n-6}},
$$

where $c_{H}$ and $\lambda_{n}$ are dimensionless parameters and $n$ is an integer greater than 4 . The first term $V_{I} \sim g^{2} M^{4}$ denotes the inflaton potential caused by the trigger field, which is displaced from the true minimum to the origin. The terms proportional to $\lambda_{n}$ originate from the typical supergravity action. For simplicity, we consider the trigger field $\chi$, the typical interaction given by

$$
\mathcal{L}_{I}=-\frac{1}{2} g^{2} \phi^{2} \chi^{2}
$$

and the decay rate $\Gamma_{\chi}=\frac{m_{\chi}^{3}}{M_{p}^{2}}>H$.

Since the decay into light matter $(\chi \rightarrow 2 \psi)$ is effective during $\phi>\phi_{*}$, the dissipative coefficient of the inflaton, which is mediated by the heavy trigger field $\chi$, is given by 2$]$

$$
\Gamma \sim 10^{-2}\left(\frac{m_{\chi}}{M_{p}}\right)^{2} \phi,
$$


where $g \sim O(1)$ is assumed 2 The strength of the damping is measured by $r$, given by the dissipative coefficient and the Hubble parameter:

$$
r \equiv \frac{\Gamma}{3 H}
$$

which can be used to rewrite the field equation of the dissipating field as

$$
\ddot{\phi}+3 H(1+r) \dot{\phi}+V(\phi, T)_{\phi}=0 \text {. }
$$

For the dissipation caused by gravitational decay, the ratio is given by

$$
r \sim 10^{-3} \times c_{r} \frac{\phi^{3}}{H M_{p}^{2}},
$$

where $c_{r}$ is a dimensionless constant 3 Note that for the modest values of $\phi<M_{p}$ and $c_{r} \sim 1$, strong dissipation is not possible if the Hubble parameter is large $\left(H \geq 10^{-3} M_{p}\right)$ during inflation. We thus implicitly assume inflation of the scale $H \ll 10^{-3} M_{p}$. From the above equations, we find that strong dissipation defined by $r>1$ is possible due to gravitational decay $\chi \rightarrow 2 \psi$. For $c_{r} \sim 1$, the condition $r>1$ leads to a strong dissipative regime given by

$$
\phi>\phi_{c} \equiv 10 \phi_{*}
$$

Thermalization of the decay product, which is very important in warm inflation, is not always important in solving the $\eta$ problem.4 Namely, if the dissipative coefficient is calculated in the zero-temperature limit, the dissipation may occur without thermalization, although inflation is not warm in that case. If the dissipation is strong for the gravitational decay, we do not need to introduce either additional symmetry that forbids a $O(H)$ mass or an artificial interaction that leads to very efficient decay of the trigger field. To

\footnotetext{
${ }^{2}$ For the decay rate $\Gamma_{\chi}=k m_{\chi}$, the dissipative coefficient is given by $\Gamma \simeq 10^{-2} g^{2} k m_{\chi}[2]$.

${ }^{3}$ Here we consider the most stringent situation with $c_{r} \sim 1$. Namely, we do not consider a situation with many light matter fields, which may enhance the decay rate by a factor of $n>10^{2}$, where $n$ is the number of light fields. The Planck-scale suppression can be relaxed if the cut-off scale appears below $M_{p}$. These effects may lead to a very large $c_{r} \gg 1$.

${ }^{4}$ In fact, massless gauge field might not exist in the very early Universe if all the gauge symmetries are completely broken by the large vacuum expectation values of the unstable flat directions. In this case, the light scalar field that remain after the symmetry breaking will be the flaton and its supersymmetric partner(the light flatino).
} 
examine more explicitly the slow-roll conditions of dissipative inflaton, we introduce new slow-roll parameters for the motion, different from the conventional slow-roll parameters. They are given by

$$
\begin{aligned}
\epsilon_{w} & \equiv \frac{\epsilon}{(1+r)^{2}}, \\
\eta_{w} & \equiv \frac{\eta}{(1+r)^{2}},
\end{aligned}
$$

where the usual slow-roll parameters $(\epsilon$ and $\eta)$ are defined by

$$
\begin{aligned}
\epsilon & \equiv \frac{M_{p}^{2}}{2}\left(\frac{V_{\phi}}{V}\right)^{2}, \\
\eta & \equiv M_{p}^{2} \frac{V_{\phi \phi}}{V} .
\end{aligned}
$$

Here the subscript denotes the derivative with respect to the field. For warm evolution the third slow-roll parameter can be defined as

$$
\beta \equiv \frac{r}{(1+r)^{3}} \frac{V_{\phi}}{3 H^{2}} \frac{\Gamma_{\phi}}{\Gamma},
$$

which sometimes raises a trivial condition, depending on the model. Calculating the slowroll parameter is straightforward. If the inflaton is far from the origin, terms proportional to $\phi^{n}$ dominate the potential. Then, using $V_{I} \sim H^{2} M_{p}^{2}$ during inflation, the slow-roll parameters are given by

$$
\begin{aligned}
& \epsilon_{w} \simeq M_{p}^{2}\left(\frac{\lambda_{n} H \phi^{n-1}}{H^{2} M_{p}^{n-1}}\right)^{2} \frac{1}{(1+r)^{2}} \sim\left(\frac{10^{3} \times \lambda_{n} \phi^{n-4}}{c_{r} M_{p}^{n-4}}\right)^{2}<1 \\
& \eta_{w} \simeq \frac{\lambda_{n}(n-1) H \phi^{n-2}}{H^{2} M_{p}^{n-3}} \frac{1}{(1+r)^{2}} \sim \frac{10^{6} \times \lambda_{n}(n-1) H \phi^{n-8}}{c_{r}^{2} M_{p}^{n-7}}<1 .
\end{aligned}
$$

The condition $\epsilon_{w}<1$ leads to

$$
\begin{aligned}
& n=4 \quad \rightarrow \quad \lambda_{4}<10^{-3} \times c_{r} \\
& n>4 \rightarrow \phi<\left(10^{3} \times \frac{\lambda_{n}}{c_{r}}\right)^{-1 /(n-4)} M_{p},
\end{aligned}
$$

and the second condition $\eta_{w}<1$ leads to

$$
\begin{aligned}
& n<8 \rightarrow \phi>\phi_{\eta}^{n<8} \equiv\left(10^{6} \times \frac{\lambda_{n}(n-1) H M_{p}^{7-n}}{c_{r}^{2}}\right)^{1 /(8-n)} \\
& n=8 \rightarrow \lambda_{n}<10^{-7} \times \frac{c_{r}^{2} M_{p}}{H} \\
& n>8 \rightarrow \phi<\phi_{\eta}^{n>8} \equiv\left(10^{-6} \times \frac{c_{r}^{2} M_{p}}{\lambda_{n}(n-1) H}\right)^{1 /(n-8)} M_{p} .
\end{aligned}
$$


The simplest example is $n=4$ and $\lambda_{4}<10^{-3}$, which leads to dissipative slow-roll during $\phi>\phi_{\eta}$. We show a schematic picture in Figure 1. Introducing $\phi_{0}$ defined by

$$
\left|\phi_{0}\right| \sim\left(\frac{c_{H} H M_{p}^{n-3}}{\lambda_{n}}\right)^{1 /(n-2)},
$$

we find that the above conditions are not applicable for $\phi<\phi_{0}$, since in this region the quadratic term is more important than the higher dimensional terms. For $\phi<\phi_{0}$, the slow-roll conditions are given by

$$
\begin{aligned}
\epsilon_{w} & \simeq M_{p}^{2}\left(\frac{c_{H} \phi}{M_{p}^{2}}\right)^{2} \frac{1}{(1+r)^{2}} \sim\left(\frac{10^{3} \times c_{H} H M_{p}}{c_{r} \phi^{2}}\right)^{2}<1, \\
\eta_{w} & \simeq \frac{c_{H}}{(1+r)^{2}} \sim \frac{10^{6} \times c_{H} H^{2} M_{p}^{4}}{c_{r}^{2} \phi^{6}}<1
\end{aligned}
$$

which lead to

$$
\begin{aligned}
& \epsilon_{w}<1 \rightarrow \phi>\sqrt{10^{3} \times \frac{c_{H} H M_{p}}{c_{r}}} \\
& \eta_{w}<1 \rightarrow \phi>10 \times\left(\frac{c_{H} H^{2} M_{p}^{4}}{c_{r}^{2}}\right)^{1 / 6} .
\end{aligned}
$$

We thus find that strong dissipation is indeed possible for $\phi<\phi_{0}$. However, in contrast to standard hybrid inflation, the slow-roll does not end with the waterfall at $\phi=\phi_{e}$.

We thus find that slow-roll inflation is possible for gravitational dissipation, but the parameter space for the slow-rolling is not trivial, depending on the model. We show a

schematic picture for a successful scenario in Figure 1. The distinguishing property of this scenario is the appearance of $\phi_{1}$ that defines the end of dissipative slow-roll inflation. Note that in the usual hybrid-type inflation model, the end of inflation is defined by $\phi_{e} \equiv M^{2} / g$, where $\chi=0$ becomes unstable. In the present model, in contrast to nondissipative inflation, the dissipative slow-roll ends at $\phi=\phi_{1}$, where the dissipative slowroll parameter reaches unity. The inflaton motion is fast during $\phi_{e}<\phi<\phi_{1}$, which occurs just before reheating at $\phi=\phi_{e}$.

\section{Curvature perturbations}

In the above argument, we considered the $\eta$-problem in supersymmetric inflation with the typical hybrid-type potential, and showed that dissipative slow-roll is possible. This 


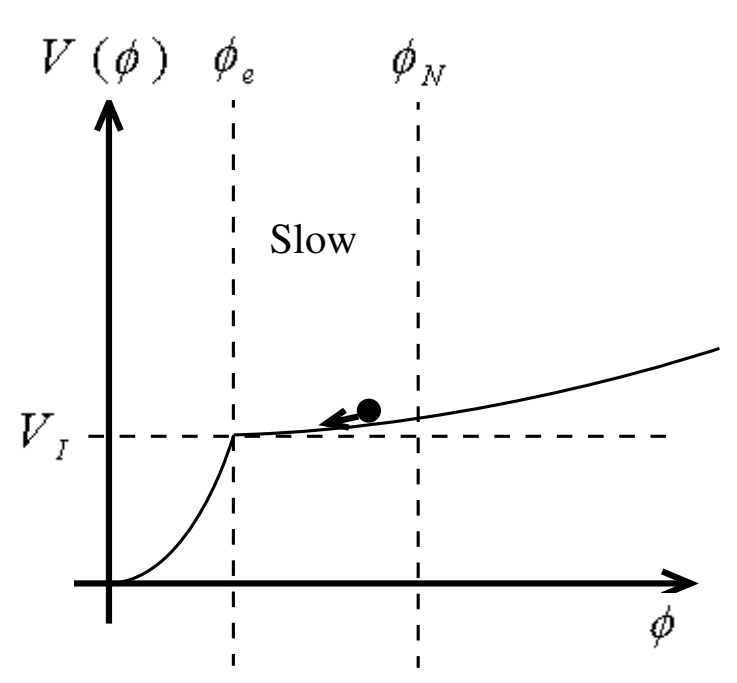

Cold Inflation

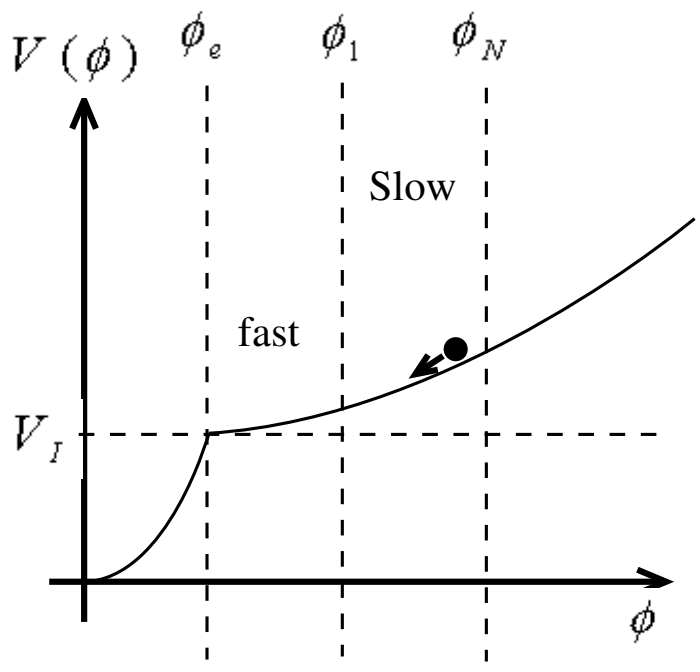

Dissipative Inflation

Figure 1: $V(\phi)$ represents the typical inflaton potential. The vacuum energy $V_{I}$ decays at $\phi=\phi_{e}$, where the trigger field starts to roll toward the true minimum. $\phi_{N}$ is defined at the horizon exit for the number of e-foldings $N_{e} . \phi_{1}$ is derived from the dissipative slow-roll conditions.

solves the traditional $\eta$ problem, though it is still not certain if the usual curvature perturbations created at the horizon exit meet the requirements of the primordial cosmological perturbations. In fact, the COBE normalization leads to 3

$$
5.3 \times 10^{-4}=\frac{r n V_{0}^{3 / 2} M_{p}^{n-6}}{\lambda_{n} H \phi_{N}^{n-1}} \simeq \frac{10^{-3} \times c_{r} n H M_{p}^{n-5}}{\lambda_{n} \phi_{N}^{n-4}} \times P_{b g}\left(r, r_{T}\right),
$$

where $P_{b g}\left(r, r_{T}\right)$ denotes the possible correction from the background radiation. Of course, $P_{b g}=1$ if there is no significant radiation background coupled to the inflaton. Here we have introduced a new parameter defined by $r_{T} \equiv T / H>1$. The source of the radiation is not specified here. From the Langevin equation, the root-mean square fluctuation amplitude of the inflaton field $\delta \phi$ after the freeze out is obtained to be

$$
\delta \phi \equiv \frac{P_{b g}\left(r, r_{T}\right) H}{2 \pi} \sim(\Gamma H)^{1 / 4} T^{1 / 2} \sim r^{1 / 4} r_{T}^{1 / 2} H \quad\left(r>1, r_{T}>1\right) .
$$

In the above argument, we assumed $\phi_{N}>\phi_{0}$. For $\phi_{N}<\phi_{0}$, if the slow-roll conditions are satisfied, the COBE normalization leads to

$$
5.3 \times 10^{-4}=\frac{r V_{0}^{3 / 2}}{M_{p}^{3} H^{2} \phi_{N}} \simeq 10^{-3} \times \frac{P_{b g}\left(r, r_{T}\right) \phi_{N}^{2}}{M_{p}^{2}} .
$$


These results depend crucially on the model. Notably, we cannot specify the temperature of the background radiation for the supersymmetric inflation model, since there are many directions in supersymmetric theory, which may dissipate and source the background [4], even though they are not the inflaton.

The above curvature perturbations, which are calculated using the standard perturbations created at the horizon crossing, may not meet the cosmological requirements. However, the mismatch does not mean that there is a crucial difficulty in the scenario. Namely, alternative scenarios for the curvature perturbations may be important for the model, as there are typically many flat directions in the supersymmetric inflation model. We thus consider in the next subsection an alternative for the generation of the curvature perturbations, which works specifically in the dissipative scenario. Other possibilities, such as inhomogeneous scenarios of phase transition[5], (p)reheating [6, 7] or curvatons [8, 9] are not discussed in this paper, because they are not characteristic of dissipative inflation. See Ref. 4] for more applications of the warm-flat directions.

\section{Modulated inflation with inhomogeneous dissipa- tion}

The inflaton potential and the interactions during inflation may be inhomogeneous due to entropy perturbations related to light fields such as moduli or flat directions in the supersymmetric model[10]. The inhomogeneities in the potential or in the interactions may lead to inhomogeneous couplings or masses that may cause fluctuations of the inflaton velocity. This is the basic idea of modulated inflation[11]. A similar situation may lead to the inhomogeneous end of hybrid inflation, as has been discussed in Ref.[12]. However, in the present scenario the inflaton velocity at the end of inflation is rapid (i.e., $\epsilon_{w} \sim 1$ or $\eta_{w} \sim 1$ at the end of inflation) and the perturbations caused by the end-boundary are less significant than the typical cosmological perturbations. On the other hand and contrary to the conventional (cold) hybrid model, the perturbations caused by the modulated

inflaton velocity $(\delta \dot{\phi})$ can be significant in the dissipative inflation model. In this section, we examine the scenario of modulated inflation in the dissipative inflation model. 
For a typical example, we consider the $S$-dependence of the dissipative coefficient $\Gamma \propto[g(S)]^{3}$ and the corrections suppressed by the cut-off scale:

$$
g(S) \equiv g_{0}\left(1+a_{1} \frac{S}{M_{*}}+\ldots\right),
$$

where $S$ is a light field that may be warm during inflation[4]. It is possible to assume a hierarchy between the cut-off scale $M_{*}$ and the Planck scale $\left(M_{*} \ll M_{p}\right)$. Note that in the usual cold model, in which the dissipation is negligible and $\chi=0$ during inflation, $\delta g$ cannot cause fluctuations of the inflaton velocity. In contrast to the cold model, in the dissipative inflation model $\delta r / r \neq 0$ caused by $\delta g$ can lead to significant inhomogeneities in the inflaton velocity. Inhomogeneous dissipation $(\delta r / r \neq 0)$ creates curvature perturbations given by 11

$$
\delta N \sim \int \frac{\delta\left(\dot{\phi}^{2}\right)}{\dot{\phi}^{2}} d t \sim 2 N_{e} \frac{\delta r}{r} \sim 6 a_{1} N_{e} \frac{\delta S}{M_{*}} .
$$

Here the flat direction $S$ may obtain $O(H)$ mass from the supergravity, but it still can move slowly if the dissipation is significant for the flat direction [4]. Also, for the warm-flat direction $S$, the amplitude of the perturbation is given by

$$
\delta S \sim\left(\Gamma_{S} H\right)^{1 / 4} T^{1 / 2} \sim r_{S}^{1 / 4} r_{T}^{1 / 2} H
$$

which shows that the amplitude is characterized by its interaction. Here we considered strong dissipation $r_{S} \equiv \Gamma_{S} / 3 H>1$ for the light field $S$. The amplitude of the warm direction is always larger than the amplitude of the cold-flat direction. The source of the background radiation is not specified, since many directions in the supersymmetric theory may dissipate during inflation and contribute to the background radiation. The spectral index of the curvature perturbation is usually determined by the potential and the interaction of the light field $S$, but for dissipative inflation it may also depend on the background radiation which may be sourced from the other flat directions.

\section{Conclusions and discussion}

In this paper we considered a dissipative inflation model for conventional supergravity using the typical hybrid-type potential. If there is no symmetry that forbids Hubbleinduced mass, the inflaton mass will be as large as the Hubble scale during inflation. This 
is the famous $\eta$ problem of the supergravity inflation model. The purpose of this paper is to find a natural solution to the problem without introducing symmetries or artificial interactions that may ruin the simplicity of the original hybrid inflation. In order to solve the problem within the naive setup, we considered only the gravitational decay mode (the gravitational decay of the trigger field into light matter) and find that the dissipation of the inflaton may be large and can dominate the friction of the inflaton motion. Then we showed that dissipative damping can provide a solution to the traditional $\eta$ problem. The dissipative inflation scenario is of course not the same as the cold scenario. A significant discrepancy that characterizes the dissipative scenario is the way the inflation ends. In the usual cold model, inflation ends with the so-called "waterfall" of the trigger field at $\phi=\phi_{e}$, while in the dissipative model slow-roll ends before the waterfall. The differences in the dynamics of the inflaton motion lead to differences in the inflationary scenario, especially for the generation of curvature perturbations. Considering the perturbations of the dissipative coefficient, we found that modulated inflation can create significant curvature perturbations.

\section{Acknowledgments}

We wish to thank K.Shima for encouragement, and our colleagues at Tokyo University for their kind hospitality.

\section{References}

[1] A. Berera, "Warm Inflation," Phys. Rev. Lett. 75, 3218 (1995) arXiv:astro-ph/9509049. A. Berera, "The warm inflationary universe," Contemp. Phys. 47, 33 (2006) arXiv:0809.4198 [hep-ph]].

[2] M. Bastero-Gil and A. Berera, "Determining the regimes of cold and warm inflation in the SUSY hybrid model," Phys. Rev. D 71, 063515 (2005) arXiv:hep-ph/0411144.

[3] A. R. Liddle and D. H. Lyth, "Cosmological inflation and large-scale structure," Cambridge, UK: Univ. Pr. (2000) 400 p. 
[4] T. Matsuda, "Aspects of warm-flat directions," arXiv:0908.3059 [hep-ph]; T. Matsuda, "Remote Inflation as hybrid-like sneutrino/MSSM inflation," arXiv:0905.4328 [hep-ph]; T. Matsuda, "Remote Inflation: Hybrid-like inflation without hybrid-type potential," JCAP 0907, 003 (2009) arXiv:0904.2821 [astro-ph.CO]]; T. Matsuda, "Evolution of the curvature perturbations during warm inflation," JCAP 0906, 002 (2009) [arXiv:0905.0308 [astro-ph.CO]].

[5] T. Matsuda, "Cosmological perturbations from an inhomogeneous phase transition," Class. Quant. Grav. 26, 145011 (2009) [arXiv:0902.4283 [hep-ph]]; T. Matsuda, "Cosmological perturbations from inhomogeneous preheating and multi-field trapping," JHEP 0707, 035 (2007) arXiv:0707.0543 [hep-th]].

[6] E. W. Kolb, A. Riotto and A. Vallinotto, "Curvature perturbations from broken symmetries," Phys. Rev. D 71, 043513 (2005) |arXiv:astro-ph/0410546]; T. Matsuda, "Generating the curvature perturbation with instant preheating," JCAP 0703, 003 (2007) arXiv:hep-th/0610232; T. Matsuda, "Generating curvature perturbations with MSSM flat directions," JCAP 0706, 029 (2007) arXiv:hep-ph/0701024.

[7] T. Matsuda, "Hybrid Curvatons from Broken Symmetry," JHEP 0709, 027 (2007) arXiv:0708.4098 [hep-ph]]; T. Matsuda, "NO Curvatons or Hybrid Quintessential Inflation," JCAP 0708, 003 (2007) [arXiv:0707.1948 [hep-ph]].

[8] A. D. Linde and V. F. Mukhanov, "Nongaussian isocurvature perturbations from inflation," Phys. Rev. D 56, 535 (1997) arXiv:astro-ph/9610219]; D. H. Lyth and D. Wands, "Generating the curvature perturbation without an inflaton," Phys. Lett. B 524, 5 (2002) arXiv:hep-ph/0110002; T. Moroi and T. Takahashi, "Effects of cosmological moduli fields on cosmic microwave background," Phys. Lett. B 522, 215 (2001) [Erratum-ibid. B 539, 303 (2002)] arXiv:hep-ph/0110096.

[9] T. Matsuda, "Curvaton paradigm can accommodate multiple low inflation scales," Class. Quant. Grav. 21, L11 (2004) arXiv:hep-ph/0312058; T. Matsuda, "Topological curvatons," Phys. Rev. D 72, 123508 (2005) arXiv:hep-ph/0509063; T. Matsuda, "Hilltop Curvatons," Phys. Lett. B 659, 783 (2008) [arXiv:0712.2103 [hep-ph]]. 
[10] L. Kofman, "Probing string theory with modulated cosmological fluctuations," arXiv:astro-ph/0303614.

[11] T. Matsuda, "Evolution of curvature perturbation in generalized gravity theories," Class. Quant. Grav. 26, 145016 (2009) [arXiv:0906.0643 [hep-th]]; T. Matsuda, "Delta$\mathrm{N}$ formalism for the evolution of the curvature perturbations in generalized multi-field inflation," arXiv:0906.2525[hep-th]; T. Matsuda, "Modulated Inflation," Phys. Lett. B 665, 338 (2008) [arXiv:0801.2648 [hep-ph]]; T. Matsuda, "Successful D-term inflation with moduli," Phys. Lett. B 423, 35 (1998) arXiv:hep-ph/9705448.

[12] F. Bernardeau, L. Kofman and J. P. Uzan, "Modulated fluctuations from hybrid inflation," Phys. Rev. D 70, 083004 (2004) [arXiv:astro-ph/0403315]; D. H. Lyth, "Generating the curvature perturbation at the end of inflation," JCAP 0511, 006 (2005) arXiv:astro-ph/0510443; T. Matsuda, "Elliptic inflation: Generating the curvature perturbation without slow-roll," JCAP 0609, 003 (2006) arXiv:hep-ph/0606137. 